\title{
Relações entre câmbio e mudança estrutural ${ }^{\dagger}$
}

Luciano Nakabashi*

RESUMO - Episódios prolongados de apreciações cambiais tendem a prejudicar o processo de crescimento puxado pela demanda externa, além de alterar a estrutura produtiva da economia, com consequências no desempenho econômico. Considerando essas relações macroeconômicas, o presente estudo tem como objetivo analisar os impactos do câmbio sobre a estrutura da economia, entre 1985 e 2008.

Palavras-chave: Exportações. Conta corrente. Taxa de câmbio real. Mudança estrutural. Desempenho econômico.

\section{INTRODUÇÃO}

Episódios prolongados de apreciação da taxa de câmbio tendem a piorar o desempenho do setor exportador, o que prejudica o crescimento devido ao problema da restrição externa, além de alterar a estrutura produtiva da economia. Se os setores mais prejudicados pela valorização cambial forem justamente aqueles mais dinâmicos, os impactos adicionais sobre o crescimento de longo prazo serão negativos. Assim, o processo de valorização cambial tende a abortar o crescimento econômico tanto pelos efeitos sobre o setor externo da economia quanto pelas mudanças em sua estrutura produtiva.

O presente estudo visa avaliar o impacto do câmbio nas mudanças estruturais da economia brasileira, sendo este realizado em duas partes. Primeiramente, fez-se uma estimação da relação entre crescimento do resto do mundo, taxa real de câmbio e exportações de produtos básicos, semimanufaturados e manufaturados, com dados trimestrais entre 1980 e 2009. Posteriormente foram considerados os coeficientes de correlação entre câmbio e estrutura do emprego formal, entre 1985 e 2008 . Nesta não foram realizadas estimações econométricas pela pequena quantidade de observações.

Além da presente introdução, contamos com uma revisão da bibliografia na segunda seção, sendo esta pela metodologia e os dados utilizados, enquanto que, na quarta seção, realizamos a análise empírica e discutimos os resultados encontrados.

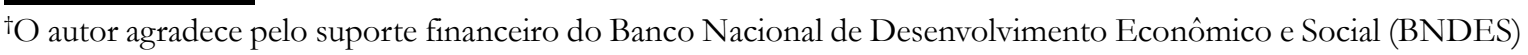
e da Coordenação de Aperfeiçoamento de Pessoal de Nível Superior (CAPES).

*Doutor em economia pelo CEDEPLAR/UFMG. Coordenador do Boletim Economia \& Tecnologia e professor adjunto do Departamento de Economia da Universidade Federal do Paraná. Endereço eletrônico: luciano.nakabashi@ufpr.br.
} 


\section{REVISÃO BIBLIOGRÁFICA}

Episódios de apreciação da taxa de câmbio, por um considerável período de tempo, afetam o crescimento à medida que essa apreciação leva a uma mudança na pauta de exportação e na estrutura produtiva de uma determinada economia.

O setor exportador reflete quais os setores da economia são mais competitivos. Desse modo, mudanças em sua composição causam alterações na estrutura produtiva de uma economia o que, por sua vez, provoca mudanças em seu desempenho dependendo do grau de dinamismo e encadeamento dos segmentos que estão perdendo e dos que estão ganhando participação.

Isso justifica a preocupação de muitos analistas econômicos com as mudanças estruturais pela qual a economia brasileira e a de outros países da América Latina vêm passando, com perda de participação relativa de setores mais dinâmicos - como a indústria - no PIB e no emprego, principalmente a partir de meados dos anos 80. Alguns exemplos são os estudos realizados por Meyer e Paula (2009), Marconi (2008), Cruz et al. (2007), Scatolin et al. (2007), Palma (2005), Feijó, Carvalho e Almeida (2005) e Bresser e Nakano (2003).

Os argumentos teóricos da importância da indústria sobre o crescimento foram apresentados por Kaldor (1957) e Hirschman (1958). O primeiro autor ressalta que a realização de investimentos na indústria leva a uma melhora do nível de tecnologia. Isso acontece porque, em muitos casos, há uma inovação tecnológica incorporada nas novas máquinas e equipamentos e esse fenômeno é ainda mais importante no setor industrial. $\mathrm{O}$ autor ainda enfatiza as economias de escalas dinâmicas geradas pelo setor industrial. Estas correspondem ao processo de aprendizado gerado na manufatura/indústria pelo ganho de experiência (learning by doing). Assim, segundo Kaldor (1957), quanto maior a taxa de crescimento da produção do setor industrial, maior também será a taxa de crescimento da sua produtividade. Hirschman (1958) aponta a importância das maiores externalidades positivas dos investimentos industriais e da importância dos efeitos de encadeamento desse setor.

No caso da economia brasileira, Chagas (2004 citado por Silva e Silveira Neto, 2007), em um estudo para os municípios paulistas, encontra evidências que sustentam os argumentos teóricos de Murphy, Shleifer e Vishny (1989), ou seja, de existência:

...de retornos crescentes de escala para setores tradicionalmente mais dinâmicos, tais como indústrias, construção civil, transporte e comunicação, serviços tecnológicos e outras atividades. Ao passo que, retornos constantes estão presentes nos setores tradicionalmente tidos como atrasados, tais quais a agropecuária, prestação de serviços e administração. (p. 2). 
Efeitos positivos no crescimento dos estados brasileiros, no período de 1994 a 2002, provenientes de efeitos de encadeamento para frente e para trás gerados pela indústria foram encontrados por Silva e Silveira Neto (2007). Feijó, Carvalho e Rodriguez (2003) apontam ainda para a importância que a indústria tem no processo de inovação e consequentemente no aumento de produtividade. Em um estudo sobre a indústria brasileira, no período de 1985-1998, eles chegaram à conclusão de que o aumento da concentração industrial levou a um aumento da produtividade do setor através do estímulo à geração de inovações.

Assim, as evidências disponíveis do papel da estrutura na economia brasileira, sobretudo do efeito do crescimento da indústria no crescimento do PIB, sugerem a existência de importantes efeitos de encadeamento da indústria com ela mesma e com outros setores, da existência de retornos crescentes de escala, além do maior potencial de inovações neste quando se compara com os demais setores da economia. Ou seja, as evidências sugerem que a estrutura produtiva da economia brasileira é um elemento relevante no seu desempenho.

\section{METODOLOGIA E FONTE DOS DADOS}

A Taxa de Câmbio Efetiva Real (TCER), do IPEA¹, é utilizada para avaliar os efeitos do câmbio na estrutura da economia. Essa variável foi utilizada porque reflete melhor o câmbio real da economia brasileira ao levar em conta diversos parceiros comerciais em seu cálculo, além de estar disponível no período de análise: 1980-2009.

Também foi utilizada a base de dados da Relação Anual de Informações Sociais (RAIS) do Ministério do Trabalho e do Emprego (MTE) para mensurar o emprego dos diferentes segmentos da economia brasileira e suas variações, entre 1985-2008, período em que os dados estão disponíveis. A base não conta com o ano de 1989 devido à falta de coleta dos dados para o mesmo. A trajetória do emprego nos diferentes segmentos da economia brasileira é utilizada para mensurar as mudanças estruturais experimentadas por esta, no período em questão. Essa análise foi feita apenas com coeficientes de correlação devido ao tamanho da amostra ser pequeno para a realização de análise de regressão. Assim, é uma primeira tentativa no sentido de se ter uma ideia entre a complexa relação das variáveis citadas.

1 A TCER é uma medida "da competitividade das exportações brasileiras calculada pela média ponderada do índice de paridade do poder de compra dos 16 maiores parceiros comerciais do Brasil. A paridade do poder de compra é definida pelo quociente entre a taxa de câmbio nominal (em R \$/unidade de moeda estrangeira) e a relação entre o Índice de Preço por Atacado (IPA) do país em caso e o Índice Nacional de Preços ao Consumidor (INPC/IBGE) do Brasil. As ponderações utilizadas são as participações de cada parceiro no total das exportações brasileiras em 2001." (Site do IPEA). 


\section{CÂMBIO E MUDANÇA ESTRUTURAL}

Com o intuito de se ter uma ideia dos efeitos do câmbio, utilizamos dados mais desagregados para a estrutura produtiva da economia brasileira, utilizando dados da RAIS/MTE para o número de empregados formais. No entanto, pelo reduzido número de observações, não seria adequado utilizar métodos econométricos para estimação. Assim, como uma primeira aproximação, utilizamos análise de correlação entre as variáveis, deixando para futuros estudos uma análise mais detalhada dessa relação.

$\mathrm{Na}$ Tabela 1 são apresentadas as correlações entre o nível de emprego de cada segmento da indústria e a taxa de câmbio real, no período 1985-2008. As variações no nível de emprego de cada segmento indicam, de forma implícita, a realização de investimentos realizados em períodos anteriores. Desse modo, não é necessário realizar a análise direta dos efeitos do câmbio nos investimentos de cada segmento. Adicionalmente, esses dados não estão disponíveis nesse nível de desagregação.

Correlações positivas entre essas duas variáveis (em vermelho) indicam que uma elevação da taxa de câmbio (depreciação) leva a um aumento no nível de empregos formais do segmento em questão. Correlações negativas (em azul) indicam que uma apreciação cambial gera um ganho no nível de emprego formal. Desse modo, os segmentos que apresentam correlações positivas (em vermelho) são mais sensíveis a períodos prolongados de apreciações reais do câmbio.

A primeira coluna da Tabela 1 apresenta as defasagens da variável taxa de câmbio real. A relação, como apontado anteriormente, vai da apreciação cambial para alterações na competitividade externa dos diferentes segmentos de forma a beneficiar os setores menos dependentes de competição via preços, ou seja, os não comercializáveis, e daqueles que experimentaram elevação da demanda externa, com consequentes alterações no nível de investimentos. Mudanças nestes, por sua vez, alteram a estrutural da economia. No entanto, leva tempo até que variações na taxa de câmbio afetem os investimentos e estes, consequentemente, a estrutura da economia, o que explica as defasagens utilizadas, que vão de 1 até 3 anos.

Na primeira linha estão os segmentos da indústria, onde cada número corresponde a um segmento, conforme nota da tabela. Observe ainda que coeficientes de correlação variam entre 0 e 1, mas como eles estão em porcentagem, ficam entre $0 \%$ e 100\%. Na última linha, encontram-se as somas dos efeitos defasados.

Os resultados apresentados na Tabela 1 evidenciam que mesmo quando não se consideram as defasagens, apreciações cambiais têm efeitos negativos no emprego industrial, de forma geral. Adicionalmente, quando se leva em conta os efeitos defasados do câmbio, notamos 
que as correlações se tornam maiores, explicando mais as variações nos respectivos níveis de emprego. Esses resultados não surpreendem, visto que a indústria é composta por segmentos de bens comercializáveis, onde a concorrência via preços é importante.

De acordo com a soma dos efeitos defasados, apresentados na última linha, os segmentos da indústria que mais sofrem, em termos de empregos, com processos de apreciação cambial real são: da madeira e mobiliário; têxtil do vestuário e artefatos de tecidos; e mecânica extrativa mineral.

TABELA 1 - COEFICIENTES DE CORRELAÇ̃̃O ENTRE NÍVEL DE EMPREGO FORMAL DOS SEGMENTOS DA ECONOMIA BRASILEIRA E TAXA REAL DE CÂMBIO REAL EFETIVA: 1985-2008

\begin{tabular}{l|rr|r|r|r|r|r}
\hline \multicolumn{1}{c|}{ Def } & \multicolumn{1}{c|}{$\mathbf{2 . 1}$} & \multicolumn{1}{c|}{$\mathbf{2 . 2}$} & $\mathbf{2 . 3}$ & $\mathbf{2 . 4}$ & \multicolumn{1}{c|}{$\mathbf{2 . 5}$} & \multicolumn{1}{c|}{$\mathbf{2 . 6}$} & \multicolumn{1}{c}{$\mathbf{2 . 7}$} \\
\hline 0 & $8,4 \%$ & $20,9 \%$ & $8,0 \%$ & $6,7 \%$ & $-4,1 \%$ & $0,1 \%$ & $46,1 \%$ \\
1 & $21,7 \%$ & $23,7 \%$ & $16,4 \%$ & $21,9 \%$ & $9,3 \%$ & $19,8 \%$ & $42,5 \%$ \\
2 & $36,5 \%$ & $24,4 \%$ & $29,0 \%$ & $36,6 \%$ & $17,0 \%$ & $37,4 \%$ & $28,0 \%$ \\
3 & $56,4 \%$ & $55,5 \%$ & $57,1 \%$ & $59,6 \%$ & $34,6 \%$ & $60,4 \%$ & $54,6 \%$ \\
Soma & $114,5 \%$ & $103,7 \%$ & $97,1 \%$ & $118,1 \%$ & $60,9 \%$ & $117,6 \%$ & $125,2 \%$ \\
\hline
\end{tabular}

\begin{tabular}{l|r|r|r|r|r|r}
\hline \multicolumn{1}{c|}{ Def } & \multicolumn{1}{c|}{$\mathbf{2 . 8}$} & \multicolumn{1}{c|}{$\mathbf{2 . 9}$} & $\mathbf{2 . 1 0}$ & $\mathbf{2 . 1 1}$ & \multicolumn{1}{c}{$\mathbf{2 . 1 2}$} & \multicolumn{1}{c}{$\mathbf{2 . 1 3}$} \\
\hline 0 & $-11,1 \%$ & $5,2 \%$ & $14,3 \%$ & $11,7 \%$ & $36,7 \%$ & $-3,7 \%$ \\
1 & $-4,1 \%$ & $16,5 \%$ & $20,7 \%$ & $27,0 \%$ & $35,3 \%$ & $2,7 \%$ \\
2 & $7,9 \%$ & $20,7 \%$ & $30,0 \%$ & $31,4 \%$ & $25,2 \%$ & $17,5 \%$ \\
3 & $59,6 \%$ & $40,6 \%$ & $62,5 \%$ & $62,2 \%$ & $49,1 \%$ & $54,1 \%$ \\
Soma & $63,4 \%$ & $77,7 \%$ & $113,2 \%$ & $120,5 \%$ & $109,7 \%$ & $74,3 \%$ \\
\hline
\end{tabular}

NOTAS: elaboração própria a partir de dados da RAIS/MTE e IPEA. 2.1 - Extrativa Mineral; 2.2 - Indústria de Produtos Minerais não Metálicos; 2.3 - Indústria Metalúrgica; 2.4 - Indústria Mecânica; 2.5 - Indústria do Material Elétrico e de Comunicações; 2.6 - Indústria do Material de Transporte; 2.7 - Indústria da Madeira e do Mobiliário; 2.8 - Indústria do Papel, Papelão, Editorial e Gráfica; 2.9 - Ind. da Borracha, Fumo, Couros, Peles, Similares, Ind. Diversas, 2.10 - Ind. Química de Produtos Farmacêuticos, Veterinários, Perfumaria; 2.11 - Indústria Têxtil do Vestuário e Artefatos de Tecidos; 2.12 - Indústria de Calçados; 2.13 - Indústria de Produtos Alimentícios, Bebidas e álcool Etílico. Def indica o número de defasagens do câmbio, sendo 1, 2 e 3 anos, enquanto Soma é a adição dos efeitos defasados $(1+2+3)$.

A Tabela 2 é análoga à anterior, com a diferença que os segmentos apresentados pertencem ao setor de serviços, à agropecuária (penúltima coluna) e ao total da economia (última coluna). Como seria de se esperar, os efeitos de variações cambiais sobre o setor de serviços não é tão acentuado quanto na indústria, pois os bens são, em geral, não comercializáveis. Os resultados apontam que alguns são beneficiados: serviços industriais de utilidade pública; construção civil; e instituições de crédito, seguros e capitalização.

Os segmentos do setor de serviços com maiores coeficientes de correlações positivos, ou seja, com maior dependência do câmbio, são: serviço de alojamento, alimentação, reparação, 
manutenção e redação; e comércio varejista. Os efeitos de apreciações cambiais também são negativos para o nível de emprego formal do setor agropecuário e da economia como um todo.

TABELA 2 - COEFICIENTES DE CORRELAÇÃO ENTRE NÍVEL DE EMPREGO FORMAL DOS SEGMENTOS DA ECONOMIA BRASILEIRA E TAXA REAL DE CÂMBIO REAL EFETIVA: 1985-2008

\begin{tabular}{l|ll|l|l|l|l|l}
\hline \multicolumn{1}{c}{ Def } & $\mathbf{3 . 1}$ & $\mathbf{3 . 2}$ & $\mathbf{3 . 3}$ & $\mathbf{3 . 4}$ & $\mathbf{3 . 5}$ & $\mathbf{3 . 6}$ & $\mathbf{3 . 7}$ \\
\hline 0 & $-52,6 \%$ & $-15,7 \%$ & $15,9 \%$ & $1,5 \%$ & $-9,1 \%$ & $20,1 \%$ & $0,7 \%$ \\
1 & $-45,8 \%$ & $-28,0 \%$ & $18,1 \%$ & $-2,4 \%$ & $-5,6 \%$ & $17,7 \%$ & $-6,7 \%$ \\
2 & $-22,7 \%$ & $-28,2 \%$ & $24,8 \%$ & $6,9 \%$ & $-2,3 \%$ & $17,2 \%$ & $1,9 \%$ \\
3 & $6,3 \%$ & $24,8 \%$ & $44,6 \%$ & $37,1 \%$ & $3,3 \%$ & $43,6 \%$ & $25,2 \%$ \\
Soma & $-62,2 \%$ & $-31,4 \%$ & $87,5 \%$ & $41,6 \%$ & $-4,5 \%$ & $78,5 \%$ & $20,4 \%$ \\
\hline
\end{tabular}

\begin{tabular}{l|c|c|c|c|c|c}
\hline \multicolumn{1}{c|}{ Def } & $\mathbf{3 . 8}$ & $\mathbf{3 . 9}$ & $\mathbf{3 . 1 0}$ & $\mathbf{3 . 1 1}$ & $\mathbf{1}$ & \multicolumn{1}{c}{ Total } \\
\hline 0 & $24,4 \%$ & $-3,1 \%$ & $-0,1 \%$ & $16,5 \%$ & $3,6 \%$ & $11,8 \%$ \\
1 & $25,5 \%$ & $-7,5 \%$ & $-4,1 \%$ & $16,7 \%$ & $-0,9 \%$ & $11,9 \%$ \\
2 & $28,8 \%$ & $-2,3 \%$ & $2,1 \%$ & $27,0 \%$ & $-1,3 \%$ & $19,1 \%$ \\
3 & $61,2 \%$ & $12,0 \%$ & $13,8 \%$ & $39,7 \%$ & $15,7 \%$ & $46,1 \%$ \\
Soma & $115,5 \%$ & $2,2 \%$ & $11,8 \%$ & $83,4 \%$ & $13,4 \%$ & $77,1 \%$ \\
\hline
\end{tabular}

NOTAS: elaboração própria a partir de dados da RAIS/MTE e IPEA. 3.1 - Serviços Industriais de Utilidade Pública; 3.2 - Construção Civil; 3.3 - Comércio Varejista; 3.4 - Comércio Atacadista; 3.5 - Instituições de Crédito, Seguros e Capitalização; 3.6 - Com. e Administração de Imóveis, Valores Mobiliários, Serv. Técnico; 3.7 - Transportes e Comunicações; 3.8 - Serv. de Alojamento, Alimentação, Reparação, Manutenção e Redação; 3.9 - Serviços Médicos, Odontológicos e Veterinários; 3.10 - Ensino; 3.11 - Administração Pública Direta e Autárquica; 1 - Agricultura, Silvicultura, Criação de Animais, Extrativismo Vegetal; Total - Total do Emprego Formal na Economia. Def indica o número de defasagens do câmbio, sendo 1, 2 e 3 anos, enquanto Soma é a adição dos efeitos defasa$\operatorname{dos}(1+2+3)$.

Um problema em se utilizar o coeficiente de correlação entre nível de emprego formal e exportações é que nem sempre uma correlação mais elevada, positiva ou negativa, significa que o câmbio tem um efeito maior sobre determinado segmento. Por exemplo, suponha dois segmentos, A e B, e o câmbio, C. Se a relação entre eles for dada por: $A_{t}=0,1 C_{t-1}$ e $B_{t}=0,5 C_{t}$ ${ }_{1}+\varepsilon_{\mathrm{t}}$; onde $\varepsilon_{\mathrm{t}}$ é um termo de erro aleatório, podemos dizer que a coeficiente de correlação é 1 no primeiro caso, enquanto que ele é menor do que um, no segundo. Ainda assim, o efeito do câmbio é maior sobre o segmento B do que sobre o A, considerando tudo mais constante. $\mathrm{Ou}$ seja, o coeficiente de correlação diz apenas o quanto uma variável está relacionada linearmente à outra. Com o suporte da teoria econômica, poderíamos dizer que o coeficiente de correlação diz quanto uma variável é explicada pela outra, mas não a magnitude do efeito de uma sobre a outra.

Para minimizar esse problema, foram empregados coeficientes de correlação entre a participação do emprego formal de cada segmento no total da economia e o nível do câmbio real. Adicionalmente, ao utilizar a participação do emprego de cada segmento no total da eco- 
nomia, podemos ter uma ideia mais clara de como variações no câmbio afetam a sua estrutura produtiva. Os resultados são apresentados nas Tabelas 3 e 4.

$\mathrm{Na}$ Tabela 3 são apresentadas as correlações entre a participação de cada segmento da indústria no emprego total da economia e a taxa de câmbio real, no período 1985-2008. Correlações positivas entre essas duas variáveis (em vermelho) indicam que uma elevação da taxa de câmbio (depreciação) leva a um ganho de participação relativo do segmento em termos de empregos formais na economia brasileira. Correlações negativas (em azul) indicam o oposto.

Os resultados apresentados na Tabela 3 apontam que quando não se consideram as defasagens, apreciações cambiais não têm efeito na estrutura da economia. No entanto, quando se leva em conta os efeitos defasados do câmbio, notamos que, praticamente, todos os segmentos da indústria perderam participação no emprego com episódios prolongados de apreciação real da taxa de câmbio, seja a defasagem de 1, 2 ou 3 anos. As exceções são os segmentos da indústria do papel, papelão, editorial e gráfica (2.8) e indústria da madeira e mobiliário (2.7).

De acordo com a soma dos efeitos defasados, apresentados na última linha, os segmentos da indústria que mais têm perdas de participação no emprego formal total devido às variações na taxa real de câmbio são: extrativa mineral; indústria mecânica; e indústria do material de transporte. É interessante notar, pelos resultados das Tabelas 1 e 3, que apesar do câmbio ser uma variável importante na explicação da indústria de madeira e mobiliário, não podemos concluir que este perde participação no emprego total com episódios de apreciação da taxa de câmbio.

TABELA 3 - COEFICIENTES DE CORRELAÇÃO ENTRE PARTICIPAÇÃO DOS SEGMENTOS NO EMPREGO FORMAL TOTAL E TAXA REAL DE CÂMBIO REAL EFETIVA: 1985-2008

\begin{tabular}{|c|c|c|c|c|c|c|c|}
\hline Def & 2.1 & 2.2 & 2.3 & 2.4 & 2.5 & 2.6 & 2.7 \\
\hline 0 & $2,4 \%$ & $4,8 \%$ & $-3,1 \%$ & $0,4 \%$ & $-7,0 \%$ & $-8,5 \%$ & $22,7 \%$ \\
\hline 1 & $11,6 \%$ & $4,5 \%$ & $0,6 \%$ & $12,9 \%$ & $0,9 \%$ & $6,3 \%$ & $17,2 \%$ \\
\hline 2 & $23,6 \%$ & $0,3 \%$ & $3,9 \%$ & $21,2 \%$ & $4,4 \%$ & $17,8 \%$ & $-9,9 \%$ \\
\hline 3 & $29,0 \%$ & $3,7 \%$ & $-0,9 \%$ & $27,8 \%$ & $10,0 \%$ & $20,3 \%$ & $-23,4 \%$ \\
\hline Soma & $64,2 \%$ & $8,5 \%$ & $3,6 \%$ & $61,9 \%$ & $15,3 \%$ & $44,4 \%$ & $-16,2 \%$ \\
\hline
\end{tabular}

\begin{tabular}{l|l|r|r|r|r|r}
\hline \multicolumn{1}{c|}{ Def } & \multicolumn{1}{c|}{$\mathbf{2 . 8}$} & \multicolumn{1}{c|}{$\mathbf{2 . 9}$} & \multicolumn{1}{c|}{$\mathbf{2 . 1 0}$} & \multicolumn{1}{c|}{$\mathbf{2 . 1 1}$} & \multicolumn{1}{c}{$\mathbf{2 . 1 2}$} & \multicolumn{1}{c}{$\mathbf{2 . 1 3}$} \\
\hline 0 & $-26,3 \%$ & $-1,6 \%$ & $-4,1 \%$ & $-2,7 \%$ & $25,0 \%$ & $-48,4 \%$ \\
1 & $-23,4 \%$ & $5,0 \%$ & $2,6 \%$ & $8,0 \%$ & $22,6 \%$ & $-23,7 \%$ \\
2 & $-22,0 \%$ & $6,0 \%$ & $2,7 \%$ & $4,4 \%$ & $3,0 \%$ & $8,5 \%$ \\
3 & $-21,6 \%$ & $12,1 \%$ & $-1,6 \%$ & $5,0 \%$ & $2,3 \%$ & $56,1 \%$ \\
Soma & $-67,1 \%$ & $23,0 \%$ & $3,8 \%$ & $17,4 \%$ & $28,0 \%$ & $40,9 \%$ \\
\hline
\end{tabular}

NOTAS: elaboração própria a partir de dados da RAIS/MTE e IPEA. 2.1 - Extrativa Mineral; 2.2 - Indústria de Produtos Minerais não Metálicos; 2.3 - Indústria Metalúrgica; 2.4 - Indústria Mecânica; 2.5 - Indústria do Material 
Elétrico e de Comunicações; 2.6 - Indústria do Material de Transporte; 2.7 - Indústria da Madeira e do Mobiliário; 2.8 - Indústria do Papel, Papelão, Editorial e Gráfica; 2.9 - Ind. da Borracha, Fumo, Couros, Peles, Similares, Ind. Diversas, 2.10 - Ind. Química de Produtos Farmacêuticos, Veterinários, Perfumaria; 2.11 - Indústria Têxtil do Vestuário e Artefatos de Tecidos; 2.12 - Indústria de Calçados; 2.13 - Indústria de Produtos Alimentícios, Bebidas e álcool Etílico. Def indica o número de defasagens do câmbio, sendo 1, 2 e 3 anos, enquanto Soma é a adição dos efeitos defasados $(1+2+3)$.

A Tabela 4 é análoga à anterior, com a diferença que os segmentos apresentados pertencem ao setor de serviços, à agropecuária (última coluna). Como seria de se esperar, os efeitos de variações cambiais sobre a participação no emprego total da economia não são claros. Os resultados apontam que alguns são beneficiados e outros não.

Os segmentos afetados negativamente, em termos de participação no emprego formal da economia brasileira, por uma apreciação cambial são: comércio varejista; comércio e administração de imóveis, valores mobiliários e serviço técnico; além do serviço de alojamento, alimentação, reparação, manutenção e redação. Todos os demais ganham participação com esses episódios, exceto a administração pública, onde o efeito líquido é próximo de zero.

TABELA 4 - COEFICIENTES DE CORRELAÇÃO ENTRE PARTICIPAÇÃO DOS SEGMENTOS NO EMPREGO FORMAL TOTAL E TAXA REAL DE CÂMBIO REAL EFETIVA: 1985-2008

\begin{tabular}{|c|c|c|c|c|c|c|}
\hline Def & 3.1 & 3.2 & 3.3 & 3.4 & 3.5 & 3.6 \\
\hline 0 & $-44,6 \%$ & $-36,7 \%$ & $23,5 \%$ & $-25,0 \%$ & $-11,0 \%$ & $35,9 \%$ \\
\hline 1 & $-41,3 \%$ & $-52,7 \%$ & $30,4 \%$ & $-33,3 \%$ & $-14,8 \%$ & $30,5 \%$ \\
\hline 2 & $-30,5 \%$ & $-60,3 \%$ & $32,3 \%$ & $-24,2 \%$ & $\cdot-12,8 \%$ & $13,0 \%$ \\
\hline 3 & $-39,9 \%$ & $-16,1 \%$ & $37,5 \%$ & $-4,5 \%$ & $-17,4 \%$ & $31,8 \%$ \\
\hline Soma & $-111,6 \%$ & $-129,1 \%$ & $100,2 \%$ & $-62,0 \%$ & $-45,0 \%$ & $75,2 \%$ \\
\hline Def & 3.7 & 3.8 & 3.9 & 3.10 & 3.11 & 1 \\
\hline 0 & $-26,4 \%$ & $16,6 \%$ & $-16,4 \%$ & $-6,8 \%$ & $9,0 \%$ & $-6,3 \%$ \\
\hline 1 & $-41,2 \%$ & $17,3 \%$ & $-21,2 \%$ & $-11,3 \%$ & $8,8 \%$ & $-11,1 \%$ \\
\hline 2 & $-37,2 \%$ & $14,2 \%$ & $-21,8 \%$ & $-9,9 \%$ & $21,0 \%$ & $-19,6 \%$ \\
\hline 3 & $-47,6 \%$ & $22,4 \%$ & $-31,1 \%$ & $-13,3 \%$ & $-29,9 \%$ & $-21,7 \%$ \\
\hline Soma & $-126,0 \%$ & $53,9 \%$ & $-74,1 \%$ & $-34,4 \%$ & $0,0 \%$ & $-52,3 \%$ \\
\hline
\end{tabular}

NOTAS: elaboração própria a partir de dados da RAIS/MTE e IPEA. 3.1 - Serviços Industriais de Utilidade Pública; 3.2 - Construção Civil; 3.3 - Comércio Varejista; 3.4 - Comércio Atacadista; 3.5 - Instituições de Crédito, Seguros e Capitalização; 3.6 - Com. e Administração de Imóveis, Valores Mobiliários, Serv. Técnico; 3.7 - Transportes e Comunicações; 3.8 - Serv. de Alojamento, Alimentação, Reparação, Manutenção e Redação; 3.9 - Serviços Médicos, Odontológicos e Veterinários; 3.10 - Ensino; 3.11 - Administração Pública Direta e Autárquica; 1 - Agricultura, Silvicultura, Criação de Animais, Extrativismo Vegetal. Def indica o número de defasagens do câmbio, sendo 1,2 e 3 anos, enquanto Soma é a adição dos efeitos defasados $(1+2+3)$.

De uma forma geral, os resultados apresentados mostram que os investimentos dos segmentos da indústria são refreados com episódios prolongados de depreciação da taxa real de câmbio de modo a reduzir a participação do emprego destes no total da economia. Isso ocorre 
porque a indústria é composta por segmentos que produzem bens comercializáveis e que, desse modo, concorrem com bens de outros países via preço e qualidade.

O mesmo não ocorre com o setor de serviços porque este é composto por segmentos de não comercializáveis e, consequentemente, não tem muita suscetibilidade da concorrência via preços. É interessante notar que o setor primário da economia tem sido beneficiado em termos de ganho de participação no emprego total formal da economia mesmo produzindo bens comercializáveis. Não exploramos esse fenômeno no presente trabalho, mesmo sendo um tema relevante, o que será tratado em estudos futuros. No entanto, uma possibilidade para essa ocorrência é devido à competitividade natural do país na produção desses bens pela sua dotação de fatores e de elementos relacionados à demanda internacional.

É importante notar ainda que os segmentos da indústria têm uma tendência natural em perder participação no emprego total devido à maior evolução da produtividade dos mesmos quando comparados com segmentos dos setores agropecuários e de serviços. No entanto, essa tendência não justifica uma correlação negativa com a taxa de câmbio, pois isto indica que a indústria perde participação relativa no emprego total nos períodos de câmbio apreciado por longos períodos de tempo e ganha naqueles em que o câmbio se encontra depreciado de forma persistente.

\section{CONCLUSÕES}

Pela análise dos coeficientes de correlação foi possível detectar efeitos relevantes do câmbio na estrutura da economia. Os resultados indicam que os segmentos do setor de serviços não têm experimentado, de forma geral, perdas de participação no emprego formal da economia com episódios de apreciação da taxa de câmbio, pois não dependem dessa variável por serem não-comercializáveis, em sua maioria. O setor agropecuário, em geral, também não apresentou perda de participação no emprego nos episódios de apreciação persistente da taxa de câmbio.

Portanto, as mudanças estruturais causadas por esses episódios, com ganhos de participação no emprego total, principalmente dos segmentos do setor de serviços, em detrimento dos segmentos da indústria, não são benéficas se estes são mais dinâmicos e com maiores possibilidades de gerar efeitos benéficos sobre o restante da economia, como um todo. 


\section{REFERÊNCIAS}

BRESSER-PEREIRA, L.C.; NAKANO, Y. Crescimento Econômico com Poupança Externa? Revista de Economia Política, 23 (2), p.3-27.

CRUZ, M.J.V.; NAKABASHI, L.; PORCILE, G.; SCATOLIN, F. D. Uma análise do impacto da composição ocupacional sobre o crescimento da economia brasileira. Economia: Revista da Anpec, 08 (Selecta), p. 55-73.

FEIJÓ, C.A.; CARVALHO, P.G.M.; ALMEIDA, J.S.G. Ocorreu uma desindustrialização no Brasil? Texto de Discussão, Instituto de Estudos para o Desenvolvimento Industrial. Disponível em: <www.iedi.org.br>.

FEIJÓ, C.A.; CARVALHO, P.G.M.; RODRIGUEZ, M.S. Concentração Industrial e Produtividade do Trabalho na Indústria de Transformação nos anos 90: Evidências Empíricas. Economia: Revista da Anpec, 4 (1), p. 19-52.

FERREIRA, A.; CANUTO, O. Thirlwall's Law and foreign capital in Brazil. Momento Econômico, 125, p. 18-29.

HIRSCHMAN, A.O. The strategy of economic development. New Haven:Yale University Press.

KALDOR, N. A Model of Economic Growth. The Economic Journal, 67 (268), p. 591624.

MARCONI, N. Existe doença holandesa no Brasil? Anais do XIII Encontro Nacional de Economia Política.

MEYER, T.R.; PAULA, L.F. Taxa de câmbio, exportações e balança comercial no Brasil: uma análise do período 1999-2006. Revista Análise Econômica, 51, p. 187-219.

MURPHY, K.M.; SHLEIFER, A.; VISHNY, R.W. Industrialization and the Big Push. Journal of Political Economy, 27 (5), p. 1003-1024.

PALMA, G. Quatro Fontes de "Desindustrialização" e um Novo Conceito de "Doença Holandesa". Trabalho apresentado na Conferência de Industrialização, Desindustrialização e Desenvolvimento. Organização: FIESP e IEDI. Local: Centro Cultural da FIESP.

SCATOLIN, F. D., CRUZ, M. J. V., PORCILE, G., NAKABASHI, L. Desindustrialização? Uma análise comparativa entre Brasil e Paraná. Indicadores FEE, 35, p. 105-120.

SILVA, M.V.B; SILVEIRA NETO, R.M. Crescimento do Emprego Industrial no Brasil e Geografia Econômica: Evidências para o Período Pós-Real. Economia: Revista da Anpec, 08 (2), p. 269-288. 